\section{G517 A NEW SPECIALIST APPROACH TO DEALING WITH PAEDIATRIC DIABETES IN THE COMMUNITY - THE HILLINGDON HOSPITAL PAEDIATRIC DIABETES INNOVATIVE SCHOOL MDT CLINICS}

S Andreou, N Williams, H Baynes, J Ganapathi. Paediatrics, The Hillingdon Hospital, London, UK

10.1136/archdischild-2015-308599.470

Background/purpose Traditionally, children and young people with Diabetes (CYPD) attend hospital clinics at least 4 times a year to see the Diabetic Multidisciplinary Team (MDT) for their regular reviews. Attendances in those patients who are in secondary school in the 11-16 year age group have been very poor at Hillingdon Hospital during 2012/13. There are multiple factors that can influence clinic non-attendance including the CYPD being reluctant to come to hospital due to the perceived burden of illness and the need for time off school during a critical period (GCSEs exams) as well as parental factors such as the need for time off work and practicalities such as difficult parking. To address these issues we decided to develop an innovative clinic model based on taking the MDT clinic out to local schools.

Material/Methods The Paediatric Diabetes MDT underwent 3 monthly visits to 4 schools in the Hillingdon borough that had 5 or more CYPD enrolled between April 2013 and the present day. The team included a Paediatric Diabetes Consultant, Specialist nurse and Dietician. Outcomes measured included number of missed appointments, patient quality of life and parents' satisfaction measured using Patient Reported Experience Measures (PREMs).

Results Our innovative approach to dealing with CYPD MDT reviews revealed: a significant reduction in missed appointments from $30 \%$ to only $2 \%$, improved patient quality of life and parent satisfaction and decreased admissions to hospital with diabetes related complications. Awareness of diabetes in school welfare officers was increased during the project.

Conclusion Our innovative approach to improving Diabetes MDT clinic attendance has had a positive impact on patients' health and quality of life. This approach could be rolled out across the UK to improve both the health and educational attainment of this vulnerable group of children.

\section{G518 ABSTRACT WITHDRAWN}

\section{G519 A NOVEL THREE-TIERED APPROACH TO IMPROVING PAEDIATRIC EDUCATION FOR GENERAL PRACTICE DOCTORS AND TRAINEES}

${ }^{1} \mathrm{C}$ Chase, ${ }^{1} \mathrm{~J}$ Edelman, ${ }^{2} \mathrm{~J}$ Barid, ${ }^{2} \mathrm{~N}$ Brown, ${ }^{2} \mathrm{~T}$ Farnon, ${ }^{1} \mathrm{~K}$ Sykes, ${ }^{3} \mathrm{~K}$ Pryde. ${ }^{1}$ Paediatric Intesive Care Unit, University Hospitals Southampton Trust, Southampton, UK; ${ }^{2}$ Paediatric Department, Salisbury District Hospital, Salisbury, UK; ${ }^{3}$ Paediatric Department, University Hospital Southampton Trust, Southampton, UK

\subsection{6/archdischild-2015-308599.471}

Context Recent recommendations from the $\mathrm{RCPCH}$ and the RCGP have highlighted the need for General Practice curricula to include more focused paediatric training.

Problem Children comprise 40\% of a typical GP's workload yet only $55 \%$ of GP trainees undertake hospital-based paediatric placements.
Assessment of problem and analysis of its causes We carried out a questionnaire of GP trainees in our region to assess confidence with Paediatrics and interest in focused paediatric education.

Intervention We hypothesised a novel three-tiered education programme would address this imbalance.

Study design We designed a curriculum-mapped educational programme for qualified GPs and GP trainees. The programme focuses on acute Paediatrics and is delivered using a variety of modalities:

Strategy for change

1. CCAP: A Crash Course in Acute Paediatrics - 2 day course for GP trainees: an overview of acute Paediatrics.

2. STrAP: Simulation Training in Acute Paediatrics - in-situ simulation for GP trainees during hospital paediatric placements.

3. GP Paediatric Refresher Days - Simulation for qualified GPs.

1. A Crash Course in Acute Paediatrics An intensive 2-day Crash Course in Acute Paediatrics (CCAP). Delivered by Paediatric registrars and nurses, this uses tutorials, workshops, skills stations and simulation to cover essential knowledge and skills. It offers an overview of assessment, and common presentations, as well as the opportunity to consolidate knowledge with relevant practical skills. The course has a strong focus on effective assessment, initial management within a primary care setting and safe transfer of patients to secondary care.

2. STrAP: Simulation Training in Acute Paediatrics A simulation programme covering common acute paediatric presentations delivered to trainees weekly and run real-time in ward areas. Simulations utilise all members of the multidisciplinary team with high-situational fidelity being achieved through use of a manikin and visual media. This gives trainees realistic exposure to assessment, diagnosis and management of an acutely unwell child.

3. GP Refresher days A Paediatric simulation programme delivered to qualified GPs at regional Refresher days. Delivered by Paediatric registrars, the programme comprises acute paediatric simulations run in real-time using equipment available in a GP surgery. The focus is on assessment, initial management and communication within the primary care environment.

Measurement of improvement CCAP candidates completed feedback questionnaires immediately following the course. All candidates felt the content was appropriate for their future careers and reported increased confidence. Feedback was almost unanimously positive, with candidates enjoying the mix of theoretical and practical learning.

STrAP candidates completed confidence questionnaires after scenarios and a basic knowledge questionnaire before and after their placements. The same questionnaires were completed with a control group of candidates not undergoing regular simulationtraining. Our results showed candidate's confidence scores increased by an average of 2 points (Likert scale 1-5) following simulation-training. Overall confidence scores reached a higher plateau 6-8 weeks sooner than the control group. Knowledge assessment results showed greater appreciation of non-specific presenting features of acute illness in the intervention group, and clearer ability with managing acute paediatric illness.

Feedback from the pilot GP simulation refresher day showed that $100 \%$ of candidates agreed or strongly agreed that the scenarios were useful and increased confidence and knowledge of acute paediatric presentations.

Effects of changes We feel our novel three-tiered education programme and its unique cross-specialty, cross-discipline delivery has improved the ability of our qualified GPs and trainees to manage acutely unwell children. 
Lessons learnt Our programme addresses the imbalance of Paediatric experience in GP training when compared to the Paediatric proportion of a typical GP's workload.

Message for others We feel the programme would benefit to GP trainees across the country and could easily be introduced in other deaneries.

\section{G520(P) WITHDRAWN}

\section{G521(P) BABY FRIENDLY PREVENTION AND MANAGEMENT OF NEONATAL HYPOGLYCAEMIA ON POSTNATAL WARDS}

${ }^{1} \mathrm{C}$ Matthews, ${ }^{2} \mathrm{E}$ Sage, ${ }^{3} \mathrm{P}$ Reynolds. 'Paediatric Surgery, Evelina Children's Hospital, London, UK; ${ }^{2}$ Paediatrics, The Royal Marsden Hospital, London, UK; ${ }^{3}$ Neonatal Intensive Care Unit, St Peter's Hospital, London, UK

10.1136/archdischild-2015-308599.472

Context The project was undertaken at a level 3 NICU in a DGH in Surrey, UK, involving junior and senior doctors, midwives, nursery nurses, pharmacists and the pathology department.

Problem Staff anecdotally felt the hypoglycaemia operational threshold was too high, monitors were inaccurate, blood sugar level (BSL) tests too frequent and management inconsistent. Treatment with formula feed supplements was hindering efforts to establish breastfeeding and prolonging stays.

Assessment of problem and analysis of its causes Initial assessment involved an audit of current practice against Baby-Friendly Initiative standards and interviews with 62 multidisciplinary team members to explore concerns and collate ideas for improvement. To check equipment accuracy, 50 samples were tested for blood glucose on both the portable metres and blood gas machine, which found on average glucosmeters under-read by $0.5 \mathrm{mmol} / \mathrm{L}$ (range $0-2.0 \mathrm{mmol} / \mathrm{L}$ ). A review of literature and other UK trust's policies was carried out to identify best practice and alternatives to formula-feed supplementation. It was recognised that new equipment would need to be used for BSL monitoring and all staff would need to be trained in any policy changes.

Intervention New guidelines lowered the working definition of hypoglycaemia, limited the number of BSL tests performed by stopping routine measurement of post-feed BSLs, and changed first line treatment of moderate hypoglycaemia (BSL $>2 \mathrm{mmol} / \mathrm{L}$ ) from formula top-up to dextrose gel. A new proforma with stepby-step management plan and built in escalation was designed with the midwives. The pathology department is acquiring more accurate glucosmeters for the trust; in the meantime a "glucose only" micro-sample setting on NICU gas machine has been set up.

Study design Audit of practice pre- and post- new management guidelines was undertaken to measure the number of needle pricks each baby had, number of days each infant at risk of hypoglycaemia was monitored for, supplementation rates, the number of babies treated for hypoglycaemia who were discharged breast feeding and still doing so at 3 months, and the number of infants admitted to NICU for management of hypoglycaemia.

Strategy for change Management of hypoglycaemia guidelines were re-written and a new proforma produced within 6 months. This was sent to all Neonatal Consultants and senior midwives for review. All midwives and nursery nurses were trained by the authors and the point-of-care team in the new guidelines, how to take a capillary blood gas and how to run a glucose only sample. Audit of practice as outlined above was carried out for infants treated 6 months before and 6 months after guideline implementation, and the results fed back to the Neonatal Consultant body.

Measurement of improvement Audit of practice showed postimplementation the average number of BSL tests per baby reduced by $73 \%$ (15 to 4 pricks) and average monitoring time reduced by $52 \%$ - saving $520 \mathrm{hrs} /$ month staff time. Breastfeeding rates at 3 months post discharge have doubled and provisional data shows a $38 \%$ reduction in SCU/NICU admissions for neonatal hypoglycaemia.

Effects of changes Changes implemented meant more accurate blood sugar analysis, fewer needle pricks for infants, shorter admission time for infants at risk of hypoglycaemia, and decreased use of formula supplementation which has enabled the trust to apply for Level 3 Baby Friendly accreditation. Some senior staff were concerned the new proforma was too complicated; this was addressed through face-to-face consultation.

Lessons learnt The most important lesson was the necessity of having all members of the multi-disciplinary team involved in the project from the beginning.

Message for others Change to the status quo to improve patient care and experience is possible by quantifying a long-standing anecdotal problem and implementing evidence based practice.

\section{G522(P) IMPROVING PAEDIATRIC MALARIA CARE IN A LOW RESOURCE SETTING}

1,2S Bihari, 1,2L Waldegrave, 'P Fillie, 'R Samuels, 1,2P Gibson. 'Paediatrics, Ola During Children's Hospital, Freetown, Sierra Leone; ${ }^{2}$ Global Links Programme, Royal College of Paediatrics and Child Health, London, UK

\subsection{6/archdischild-2015-308599.473}

Context The improvement took place in a large paediatric referral hospital in West Africa.

Problem Malaria is one of the leading causes of morbidity and mortality for children attending the hospital. There was no uniform guidance or set standard on the management of malaria. As a result, treatment varied from doctor to doctor, patient to patient.

Assessment of problem and analysis of its causes 40 clinical notes per week were randomly selected for 5 weeks (Weeks 1-5). The data collected compared current practice against the standard expected for the management of a child presenting with a fever/malaria. Multiple areas where the standards were not met were identified at all stages of the child's journey from presenting to the hospital with fever all the way to discharge home or death.

Intervention A new malaria guideline and training package was developed. The guideline included a flow chart, investigations and $1^{\text {st }}$ and $2^{\text {nd }}$ line treatment options, a drug treatment table and guidance on how to prepare and prescribe the treatment on a drug chart. These guidelines were approved by the National Malaria Control Programme and then implemented across the hospital, through teaching and training workshops.

Study design The study used two cohort groups of patients; The first cohort included patients who were admitted to the hospital prior to the intervention the second cohort included patients who were admitted to the hospital after the intervention. The data analysed compared the findings between both groups. 